\title{
Leishmania LABCG1 and LABCG2 transporters are involved in virulence and oxidative stress: functional linkage with autophagy
}

José Ignacio Manzano', Ana Perea', David León-Guerrero', Jenny Campos-Salinas', Lucia Piacenza², Santiago Castanys $^{1 *+}$ and Francisco Gamarro ${ }^{1 *+}$

\begin{abstract}
Background: The G subfamily of ABC (ATP-binding cassette) transporters of Leishmania include 6 genes (ABCG1-G6), some with relevant biological functions associated with drug resistance and phospholipid transport. Several studies have shown that Leishmania LABCG2 transporter plays a role in the exposure of phosphatidylserine (PS), in virulence and in resistance to antimonials. However, the involvement of this transporter in other key biological processes has not been studied.

Methods: To better understand the biological function of LABCG2 and its nearly identical tandem-repeated transporter LABCG1, we have generated Leishmania major null mutant parasites for both genes ( $\triangle$ LABCG1-2). NBD-PS uptake, infectivity, metacyclogenesis, autophagy and thiols were measured.

Results: Leishmania major $\triangle L A B C G 1-2$ parasites present a reduction in NBD-PS uptake, infectivity and virulence. In addition, we have shown that $\triangle \mathrm{LABCG} 1-2$ parasites in stationary phase growth underwent less metacyclogenesis and presented differences in the plasma membrane's lipophosphoglycan composition. Considering that autophagy is an important process in terms of parasite virulence and cell differentiation, we have shown an autophagy defect in $\triangle$ LABCG1-2 parasites, detected by monitoring expression of the autophagosome marker RFP-ATG8. This defect correlates with increased levels of reactive oxygen species and higher non-protein thiol content in $\triangle$ LABCG1-2 parasites. HPLC analysis revealed that trypanothione and glutathione were the main molecules accumulated in these $\triangle$ LABCG1-2 parasites. The decrease in non-protein thiol levels due to preincubation with buthionine sulphoximide (a $\gamma$-glutamylcysteine synthetase inhibitor) restored the autophagy process in $\triangle$ LABCG1-2 parasites, indicating a relationship between autophagy and thiol content.

Conclusions: LABCG1-2 transporters from Leishmania could be considered as phosphatidylserine and nonprotein thiol transporters. They probably accomplish transportation in conjunction with other molecules that are involved in oxidative stress, autophagy, metacyclogenesis and infectivity processes. The overall conclusion is that LABCG1-2 transporters could play a key role in Leishmania cell survival and infectivity.
\end{abstract}

Keywords: Leishmania, ABC transporters, Virulence, Metacyclogenesis, Autophagy, Oxidative stress

\footnotetext{
*Correspondence: castanys@ipb.csic.es; gamarro@ipb.csic.es

†'Equal contributors

${ }^{1}$ Instituto de Parasitología y Biomedicina "López-Neyra", IPBLN-CSIC, Parque

Tecnológico de Ciencias de la Salud, Avda. del Conocimiento s/n, 18016

Granada, Spain

Full list of author information is available at the end of the article
} 


\section{Background}

Leishmaniasis is considered a neglected tropical disease caused by protozoan parasites of the genus Leishmania [1]. It is prevalent in 98 countries around the world and the current incidence is estimated about 0.2-0.4 million cases of visceral leishmaniasis and 0.7-1.2 million cases of the cutaneous form [1].

ABC (ATP-binding cassette) transporters are constituted by two homologous halves to be functional. The binding of substrates occurs in the transmembrane domain while the hydrolysis of ATP needed for the transport occurs in a cytosolic nucleotide binding domain [2]. The Leishmania genome contains $42 \mathrm{ABC}$ genes classified in 9 subfamilies (from ABCA to ABCI) [3, 4]. The ABCG subfamily includes half-transporters that require homo/heterodimerisation to become functional [5]. LABCG2 has two additional imperfect tandem repeats in chromosome 6 of Leishmania (LABCG1 and LABCG3) [6]. LABCG1 and LABCG2 are almost identical (93\% amino acid identity); however, the LABCG3 protein is truncated at the nucleotide binding and transmembrane domains. Expression of a dominant-negative version of the half-transporter LABCG2 produces a defect in the external surface exposure of endogenous phosphatidylserine (PS), which is normally asymmetrically confined on the inner leaflet of eukaryotic cells' plasma membranes. Additionally, these parasites present a decrease in the infection of mouse peritoneal macrophages and reduced virulence in a mouse model of cutaneous leishmaniasis [6].

The process by which trypanosomatids metabolically differentiate from procyclic promastigotes (non-infective) into metacyclic promastigotes (infective) is the metacyclogenesis [7]. In Leishmania species, the place where metacyclogenesis occurs is in the insect vector; in vitro, this process can be induced by acidification of the medium after the growth of parasites from logarithmic to stationary phase [8]. Stage-specific variations are observed throughout the parasite life-cycle, such as the considerable structural modifications to lipophosphoglycan (LPG) composition and structure during parasite metacyclogenesis. LPG plays an important role in establishing Leishmania infection by conferring resistance to lysis mediated by complement and protecting from oxidative injury, by facilitating the binding to other receptors of macrophages and by remodeling the initial phagolysosome [9-11]. To date, there are no reports of a Leishmania $\mathrm{ABC}$ transporter involved in metacyclogenesis or modification of LPG composition.

In Leishmania, autophagy is a well-conserved process required for degradation of proteins and organelles during cellular differentiation and metacyclogenesis [12]; the ATG8-lipidation pathway plays an important role for autophagosome synthesis during autophagy. Moreover, the relationships between oxidative stress, caused by high levels of reactive oxygen species (ROS), and autophagy in Leishmania have been clearly established. Also, glutathione $(\mathrm{GSH})$ is known to be involved in mitochondrial autophagy regulation in yeast [13]. Evidence that thiol pools have a modulatory function in autophagy progression due to an ABCC1-dependent extrusion has also been published [14]. The intracellular redox state of thiol pools, which markedly depends on GSH levels, could drive autophagy processes in carcinoma cells [14].

Recent studies indicate that human ABCG2 is involved in autophagy regulation and strongly suggest that ABCG2 plays a key role in cell survival [15]. Tumour cells overexpressing ABCG2 enhance both autophagy and cell survival suggesting that this transporter assumes a previously unknown role beyond its conventional drug-efflux function, probably associated with the transport of a specific cellular substance (or substances) involved in autophagy regulation [15].

On the other hand, some $\mathrm{ABC}$ transporters are able to transport thiols while conjugated with other substances. In this manner, several members of the ABCC subfamily (MRP1, MRP2, MRP3, MRP4, MRP5 and CFTR) and $\mathrm{ABCB} 7 / \mathrm{ATM} 1$ have been described as GSH transporters involved in cellular detoxification in mammalian cells $[16,17]$. Indeed, overexpression of ABCG2 in mammalian cells altered intracellular GSH levels [18], although the role this transporter plays in that modulation process is still unclear. In Leishmania, LABCG2 overexpression is involved in antimony resistance; this is mediated by a lower accumulation due to intracellular sequestration and increased antimony and thiol efflux through the parasites' flagellar pocket [19].

In order to better understand the role of LABCG1-2 transporters in Leishmania cell survival and infectivity, we have obtained null mutant parasites for both genes. Here, we report that LABCG1 and 2 not only influence infectivity, virulence and metacyclogenesis but they also regulate the rate of autophagy and redox metabolism. Taken as a whole, the data presented here suggest that Leishmania LABCG1-2 transporters have a previously unknown biological role associated with autophagy, establishing a relationship between thiol pool levels and autophagy.

\section{Methods}

\section{Animals}

BALB/c mice (six-week-old, female) from Charles River Breeding Laboratories were maintained and fed under pathogen-free conditions in the Animal Facility Service of the Instituto de Parasitología y Biomedicina "López-Neyra".

\section{Strain and culture conditions}

Leishmania major (MHOM/JL/80/Friedlin) was the chosen strain. This line and derivative lines used in this study were maintained in RPMI 1640 medium (Invitrogen, 
Paisley, UK) supplemented with $20 \%$ heat-inactivated fetal bovine serum (hiFBS, Invitrogen) at $28{ }^{\circ} \mathrm{C}$.

\section{Generation of $L$. major LABCG1 and LABCG2 null mutants} To achieve targeted gene replacement of the $L$. major LABCG1 and LABCG2 genes (GeneDB-L. major, accession codes LmjF06.0080 and LmjF06.0090, respectively), we constructed a targeting DNA fragment in which the hyg gene, which confers resistance to hygromycin B, was flanked at the $5^{\prime}$ end by a $1.5-\mathrm{kb}$ region containing the LABCG 2 stop codon and at the $3^{\prime}$ end by a $1.5-\mathrm{kb}$ region containing the LABCG1 initiation codon. The different fragments were amplified by PCR from genomic DNA using the following pairs of complementary primers: P1 forward (5'-atg cgg ccg ccg cTG TTT ATC TGT GTT ATC G-3') and P2 reverse (5' -atg gat cct cta gaa ttt aaa tGT AGA CAG GCG GAG AAG GCA G-3') for the 5' region; P3 forward ( $5^{\prime}$-att cta gaG TTG TAA GCT GCT GTG CGG CGT AAC-3') and P4 reverse (5' -atg gat ccG CAC ACG CGC GTA GGA AAG CAG-3') for the $3^{\prime}$ region; or from the cLHYG vector: P5 (5' -GTA GAT CTA CCA CTT TCT GCC TTC TG-3') and P6 (5-GGA AGC TTC TAT TCC TTT GCC CTC GGA CG-3') for the resistance cassettes. All fragments were subcloned into the pGEM ${ }^{\oplus}$-T vector (Promega, Madrid, Spain).

Leishmania major promastigotes in logarithmic phase of growth were transfected with $2 \mu \mathrm{g}$ of linearized DNA targeting constructions by electroporation Nucleofector ${ }^{\text {TM }}$ (Lonza, Köln, Germany). In the first round of selection, the electroporated promastigotes were incubated in drug-free culture medium for $24 \mathrm{~h}$, and plated in 96-well microplates in the presence of $10 \mu \mathrm{g} / \mathrm{ml}$ hygromycin B. By this method, several single knock-out clones were obtained. In a second round of gene targeting, loss of heterozygosity was promoted by increasing the hygromycin B concentration up to $500 \mu \mathrm{g} / \mathrm{ml}$ and plating into agar plates as previously described [20]. The clones obtained were analysed by Southern blot.

\section{Southern blot analysis}

Genomic DNA was purified from WT (wild-type) and LABCG1-2 null mutant ( $\triangle$ LABCG1-2) parasites and then digested with AgeI. Digested DNA was then separated using agarose gel electrophoresis, transferred on to a nylon membrane and hybridised to digoxigeninlabelled (DIG) DNA probes from an intergenic region between $L A B C G 1$ and $L A B C G 2$ (probe 1) or the $5^{\prime}$ UTR of $L A B C G 2$ (probe 2), as described by [21]. Templates for synthesis of both probes were obtained by PCR from genomic DNA of L. major using the pairs of primers $\mathrm{S} 1$ forward (5'-ATC CAC CCG TCG ACA CAT GC-3') and S2 reverse (5'-CGC TGT CCT TCC GTT TGT GG-3'), and S3 forward (5'-TGT TTA TCT GTG TTA TCG-3') and S4 reverse (5'-GTA GAC AGG CGG
AGA AGG CAG-3'), respectively. All procedures for the DIG application system (Roche, Indianapolis, USA) were carried out according to the manufacturer's instructions.

\section{Transfection of Leishmania lines}

$\triangle$ LABCG1-2 parasites were transfected and selected for resistance to $50 \mu \mathrm{g} / \mathrm{ml}$ of $\mathrm{G} 418$, when restituting $L A B C G 2$ ( $\triangle \mathrm{LABCG} 1-2+\mathrm{LABCG} 2)$, or to nourseothricin, in the case of $L A B C G 1$ ( $\triangle \mathrm{LABCG} 1-2$ + LABCG1), as previously described [6]. Double transfection techniques with the plasmids previously used for single gene reconstitution were performed to generate the add-back line $\triangle$ LABCG12 + LABCG1-2. To monitor autophagy, parasites were transfected with the plasmid pNUS RFP-ATG8 and selected at a concentration of $50 \mu \mathrm{g} / \mathrm{ml}$ of blasticidin.

\section{Analysis of fluorescent PS uptake in Leishmania lines}

Accumulation of the fluorescent lipid analogue of PS (NBD-PS, palmitoyl-2-[6-(7-nitrobenz-2-oxa-1,3-diazol-4yl) amino] hexanoyl-sn-glycero-3-phosphoserine, from Avanti Polar Lipids (Birmingham, AL, USA) was determined by flow cytometry, as described previously [6]. Briefly, stationary-phase promastigotes $\left(10^{7} / \mathrm{ml}\right)$ were incubated in HPMI buffer (20 mM HEPES, $132 \mathrm{mM} \mathrm{NaCl}$, $3.5 \mathrm{mM} \mathrm{KCl}, 0.5 \mathrm{mM} \mathrm{MgCl}_{2}, 5 \mathrm{mM}$ glucose, $1 \mathrm{mM} \mathrm{CaCl}_{2}$, $\mathrm{pH}$ 7.4) supplemented with $0.3 \%(\mathrm{w} / \mathrm{v})$ BSA (Bovine serum albumin) for $30 \mathrm{~min}$ at $28{ }^{\circ} \mathrm{C}$ and then labelled with $30 \mu \mathrm{M}$ NBD-PS for $30 \mathrm{~min}$ at $28^{\circ} \mathrm{C}$. HPMI was supplemented with either $500 \mu \mathrm{M}$ PMSF (phenylmethylsulfonyl fluoride), or $5 \mathrm{mM}$ DFP (diisopropylfluorophosphate) to block the catabolism of NBD-lipids. NBD-PS remaining on the cell surface was extracted twice by washing with 0.3\% (w/v) BSA in PBS buffer. Parasites were resuspended in PBS for flow cytometry analysis performed with a Beckton Dickinson FACScan (San José, USA) equipped with an argon laser operating at $488 \mathrm{~nm}$.

\section{In vitro infection of mouse peritoneal macrophages}

The infection assays of peritoneal macrophages from BALB/c mice (Charles River Ltd., Barcelona, Spain) were performed as described previously [4] with some modifications. Briefly, the adherent macrophages placed in 24wells plate with coverslips, were infected for $4 \mathrm{~h}$ at $35^{\circ} \mathrm{C}$ with stationary-phase promastigotes at a parasite-to-cell ratio of 2:1. The infection was stopped by washing with serum free medium and the infected macrophages were incubated for 24, 72 and $120 \mathrm{~h}$. After incubation, the cultures were processed and stained with DAPI $\left(4^{\prime}, 6-\right.$ diamidino-2-phenylindole dilactate) for the calculation of the percentage of infection and the mean number of amastigotes by fluorescence microscopy. 


\section{Analysis of in vivo infection}

Animals (6 mice/group) were injected subcutaneously in their left hind footpads with $10^{5}$ stationary-phase $L$. major promastigotes resuspended in PBS. Progression of the disease was monitored with weekly measurements of the footpad swelling, assessed by measuring the width of infected footpad, and extent of the cutaneous lesion on the infected footpad using a digital calliper (Mitutoyo, Japan), as described previously [22].

\section{Lectin-mediated agglutination of Leishmania lines}

Promastigotes were agglutinated using lectin PNA (Peanut agglutinin) in order to purify metacyclic promastigotes, as described previously [23]. Parasites $\left(1 \times 10^{8}\right)$ were collected in stationary-phase growth, washed twice with PBS and incubated with $100 \mu \mathrm{g} / \mathrm{ml}$ of PNA for $10 \mathrm{~min}$ at room temperature. The parasites were then centrifuged for $10 \mathrm{~min}$ at $500 \times g$. Finally, non-agglutinated parasites remaining in the supernatant were centrifuged, resuspended in PBS and counted with a haemocytometer to determine the total cell number.

\section{Western blot analysis}

Parasites expressing RFP-ATG8 (autophagosomal marker) were harvested at $1,000 \times g$ for $10 \mathrm{~min}$ and washed twice in PBS; the pellets were either used immediately or stored at $-20{ }^{\circ} \mathrm{C}$. The parasite lysates were obtained by resuspending parasite pellets in lysis buffer, containing $150 \mathrm{mM}$ Tris- $\mathrm{HCl}, 50 \mathrm{mM} \mathrm{NaCl}, 2 \%$ DDM (n-dodecyl- $\beta$-D-maltopyranoside) and a mixture of peptidase inhibitors (Thermo Scientific, Rockford, USA).

Promastigote cell extracts were separated by SDSPAGE (10\%) in the presence of $6 \mathrm{M}$ urea and analysed by Western blotting using an anti-RFP antibody (1:5,000; Invitrogen) as described [12]. An enhanced chemiluminescence reaction with an ECL kit (Amersham, Rockford, USA) was used for detection.

For LPG analysis, stationary-phase promastigotes (8 days of their growth cycle) were harvested at $1,000 \times g$ for $10 \mathrm{~min}$ and washed twice in PBS. Parasite lysates used for this analysis were obtained as described above. Promastigote cell extracts were separated by SDS-PAGE (12\%) and further analysed by Western blotting with the monoclonal antibodies: WIC 73.9 (1:250), specific for terminal Gal ( $\beta 1-3)$ side-chains of LPG in plasma membrane [24]; and with 3 F12 (1:500), which recognises specific arabinose residues of metacyclic forms [25].

For evaluation of metacyclogenesis, the metacyclic specific protein HASPB was analysed using rabbit antiHASPB antibody at a 1:5,000 dilution [26]. The protein 3-hydroxy-3-methyl-glutaryl Coenzyme A synthase (LmHMGS), used as an internal loading control, was recognised using rabbit anti-LmHMGS antibody at a 1:100,000 dilution [27].

\section{Measurement of ROS levels in Leishmania lines}

The levels of intracellular ROS were measured as described [12]. Briefly, promastigotes $\left(1 \times 10^{7}\right)$ were collected at early logarithmic (EL), mid logarithmic (ML), early stationary (ES) and stationary (S) phases of growth. Cells $\left(2 \times 10^{6}\right)$ were incubated with $0.1 \mathrm{mM} \mathrm{H}_{2}$ DCF-DA for $2 \mathrm{~h}$ at $28{ }^{\circ} \mathrm{C}$. Fluorescence was then measured through flow cytometry (excitation 380-420 nm, barrier filter $520 \mathrm{~nm}$ ) using a FACSCalibur ${ }^{\mathrm{Tm}}$ (Becton-Dickinson, San Jose, USA).

\section{Complement-mediated lysis in Leishmania lines}

The susceptibility of promastigotes to complementmediated lysis was assessed following the protocol previously described [28] but with some modifications. Briefly, promastigotes $\left(1 \times 10^{7} / \mathrm{ml}\right)$ in a logarithmic or stationary phase of growth were exposed to increasing concentrations of fresh human serum for $30 \mathrm{~min}$ at $37^{\circ} \mathrm{C}$. After exposure, resazurin was added and incubated at $28{ }^{\circ} \mathrm{C}$ for $24 \mathrm{~h}$ in order to discover the cell viability. Sample fluorescence (excitation: $540 \mathrm{~nm}$; emission: $580 \mathrm{~nm}$ ) was measured in an Infinite F200 Luminescence System (Tecan Austria GmbH, Grödig, Austria).

\section{Monitoring autophagy in Leishmania lines}

Promastigotes expressing the autophagosomal marker RFP-ATG8 were examined daily under a fluorescence microscope in order to assess the proportion of parasites containing autophagosomes and the number of these structures per parasite. Three series of 200 parasites were counted for each time-point in each experiment. To determine the relationship between thiol levels and autophagy in Leishmania lines, $3 \mathrm{mM}$ BSO (buthionine-[S,R]-sulfoximine, a $\gamma$-glutamylcysteine synthetase inhibitor) was added for $48 \mathrm{~h}$ before the autophagy experiments.

\section{Determination of intracellular non-protein thiol levels}

To determine intracellular thiol levels, we used the fluorimetric probe CellTracker ${ }^{\mathrm{TM}}$ as described previously [19]. Log phase parasites $\left(10^{7} / \mathrm{ml}\right)$ were grown in M-199 medium plus $10 \%$ hiFBS. They were then washed with PBS and incubated with $2 \mu \mathrm{M}$ CellTracker ${ }^{\mathrm{TM}}$ for $15 \mathrm{~min}$ at $37{ }^{\circ} \mathrm{C}$. After incubation, parasites were washed with PBS again and analysed by flow cytometry using a FACScan flow cytometer (Becton-Dickinson). Fluorescence emission between 515 and $545 \mathrm{~nm}$ was quantified using CellQuest ${ }^{\mathrm{TM}}$ software.

To determine non-protein thiol levels, mid-log phase promastigotes $\left(1 \times 10^{9}\right.$ cells) were collected by centrifugation $(1,000 \times g, 10 \mathrm{~min})$ and washed three times with PBS. Proteins were precipitated with $2 \%$ trichloroacetic acid for $30 \mathrm{~min}$ and the supernatants obtained were neutralised with Tris- $\mathrm{HCl} 1 \mathrm{M}$ at $\mathrm{pH}$ 8. We used $1 \mathrm{mM}$ of DTNB (5,5dithiobis-2-nitrobenzoic acid) for $10 \mathrm{~min}$ to measure the reduced non-protein thiol levels. The absorbance obtained at 
$412 \mathrm{~nm}$ was interpolated on a GSH standard curve generated using different concentrations of reduced GSH.

To quantify and discriminate between the different thiol molecules in the sample, the cell pellet was lyophilised and resuspended in $100 \mu \mathrm{l}$ of HEPES (40 mM, pH 8.0) containing a freshly prepared aqueous solution of $\mathrm{NaBH}_{4}$ (sodium borohydride, $100 \mathrm{mM}$ final concentration). Samples were allowed to set for $30 \mathrm{~min}$ at room temperature and thiols were then derivatized with monobromobimane $(\mathrm{mBBr}$, $2 \mathrm{mM}$ final concentration) for $5 \mathrm{~min}$ at $70{ }^{\circ} \mathrm{C}$ for detection. Following derivatisation, proteins were precipitated and any excess $\mathrm{NaBH}_{4}$ removed by acid incubation with methanesulphonic acid $(4 \mathrm{M}, 100 \mu \mathrm{l})$ for $2 \mathrm{~h}$ on ice. Acid soluble thiols were separated by reverse phase ion-pairing HPLC on an Agilent C18 column and analysed through fluorometric detection [29]. Quantitation and validation were accomplished using $\mathrm{T}[\mathrm{SH}]_{2}$ and GSH standards.

\section{Statistical analysis}

Statistical treatment of data obtained between groups were performed using the Student's $t$-test. Differences were considered significant at a level of $P<0.05$.

\section{Results and discussion}

Molecular characterisation of L. major LABCG1 and LABCG2 null mutants

Genes encoding LABCG1 and LABCG2 are tandemly linked with each other on chromosome 6 of Leishmania (LABCG1-2) (Fig. 1a) [6]. A L. major mutant lacking both LABCG1 and LABCG2 genes ( $\triangle$ LABCG1-2) was generated so we could further investigate the biological function of these proteins. The chromosomal loci containing the genes encoding LABCG1-2 were substituted with antibiotic cassettes that confer resistance to hygromycin B after applying a high selection pressure (up to $500 \mu \mathrm{g} / \mathrm{ml}$ ) with hygromycin $\mathrm{B}$ on the parasite population (Fig. 1a). In order to verify that both LABCG1-2 alleles were deleted, a Southern blot analysis was carried out on genomic DNA using different probes: the intergenic region of LABCG1-2 (probe 1, Fig. 1a, b1), and the 5' UTR LABCG2 (probe 2, Fig. 1a, b2).

Loss of LABCG1-2 results in an increase in NBD-PS uptake in Leishmania lines

After confirming the null mutant genotypes, $\triangle$ LABCG12 parasites were tested for their capacity to transport NBD-PS. As previously described, the expression of a

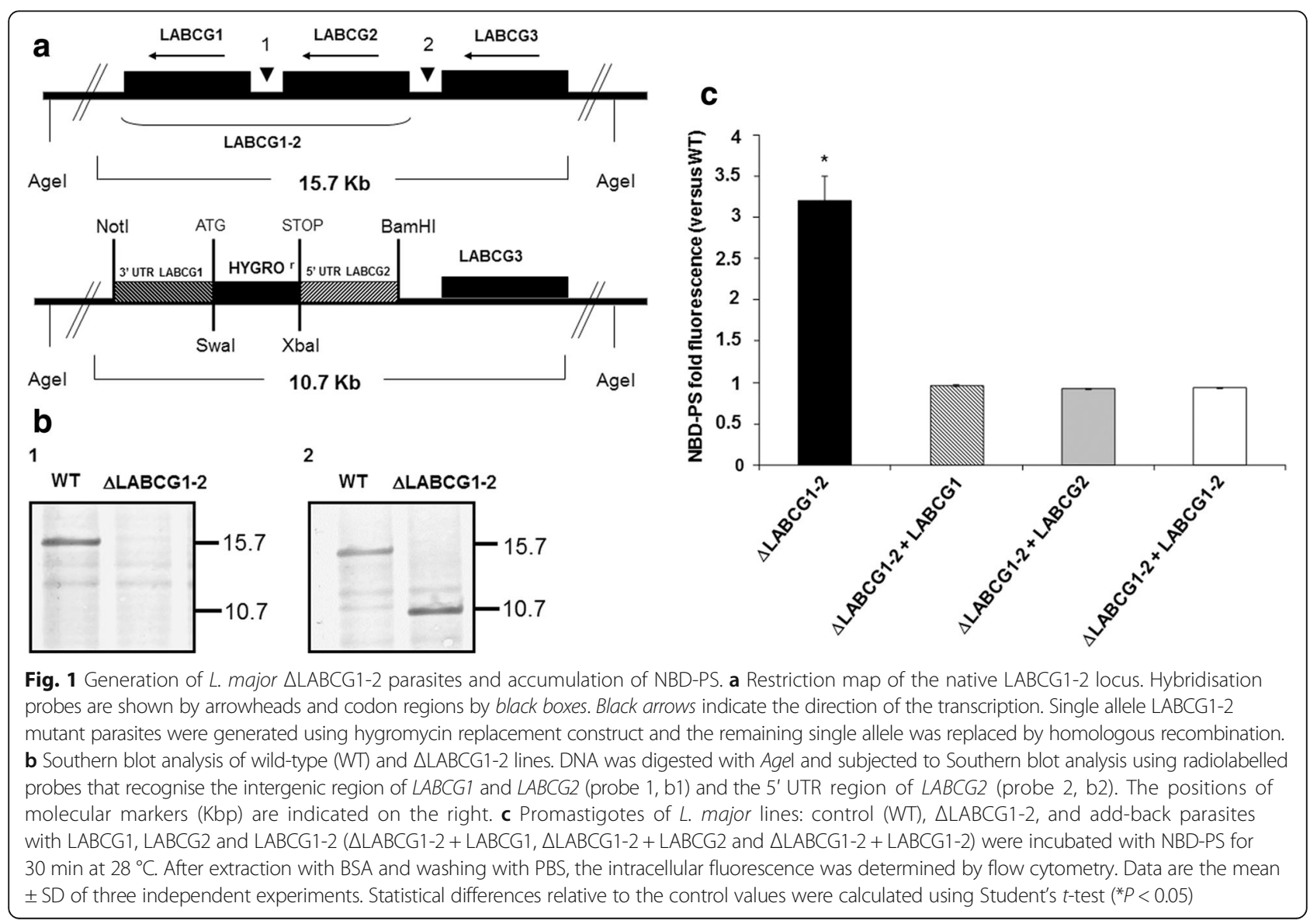


dominant-negative mutant version of LABCG2 results in a significantly higher accumulation of NBD-PS [6]. As expected, $\triangle$ LABCG1-2 parasites showed a higher accumulation of NBD-PS compared to WT and add-back parasites ( $t$-test: $t_{(4)}=12.92, P=0.0002$ for WT; $t$-test: $t_{(4)}=13.06, \quad P=0.0002$ for $\triangle \mathrm{LABCG} 1-2+$ LABCG1-2; Fig. 1c). Additionally, parasites $\triangle \mathrm{LABCG} 1-2+\mathrm{LABCG} 1$, $\triangle \mathrm{LABCG} 1-2+\mathrm{LABCG} 2$ and $\triangle \mathrm{LABCG} 1-2+\mathrm{LABCG} 1-2$ have similar NBD-PS accumulation values (Fig. 1c), supporting the suggestion that the function of LABCG1 and LABCG2 transporters is similar to the one previously considered when taking into account their high \% of amino acid identity (93\%). Thus, our results support that Leishmania LABCG1 and LABCG2 transporters have PS floppase activity. We decided to continue the functional studies using add-back parasites $\triangle$ LABCG1-2 + LABCG12 to achieve the highest possible level of similarity with the WT line, avoiding potential functional differences between LABCG1 and LABCG2 transporters.

\section{Decreased infectivity and virulence in $\triangle$ LABCG1-2 parasites}

To determine the biological effects of the genetic deficiencies in LABCG1-2 transporters on Leishmania, the infectivity and survival of WT, $\triangle$ LABCG1-2 or add-back parasites in mouse peritoneal macrophages were determined at 24, 72 and $120 \mathrm{~h}$ post-infection. The results show that $\triangle$ LABCG1-2 parasites have a lower percentage of infection (20-35\%) compared with WT and addback lines $(65-75 \%)\left(t\right.$-test: $t_{(4)}=9.14-21.02, P=0.0001-$ 0.0008 ; Fig. 2a). Additionally, the mean number of parasites per infected macrophage in $\triangle \mathrm{LABCG} 1-2$ parasites was significantly lower compared to the other lines, indicating a significantly lower parasite entry into macrophages at $24 \mathrm{~h}$ post-infection; these parasites were viable and replicate inside the macrophages at 72 and $120 \mathrm{~h}$ ( $t$-test: $t_{(4)}=4.35-36.87, P=0.0001-0.0121$; Fig. $\left.2 \mathrm{~b}\right)$.

We then analysed whether these results were linked to a lower in vivo virulence of the parasites using a mouse model of cutaneous leishmaniasis. Mice infected with WT and add-back parasites showed progressive swelling and lesions after 5 weeks (Fig. 3a-c), whereas mice infected with $\triangle \mathrm{LABCG} 1-2$ parasites presented minimal lesion pathology and significantly lower footpad swelling (Fig. 3a-c). Thus, we confirmed that LABCG1-2 are relevant transporters involved in infectivity and are essential for disease development.

\section{Defect in metacyclogenesis and changes in LPG composition in $\triangle$ LABCG1-2 parasites}

Based on the above results, we assessed whether metacyclogenesis was impaired in $\triangle$ LABCG1-2 parasites by studying several properties that distinguish metacyclic promastigotes from procyclic ones; among others, agglutination to PNA,

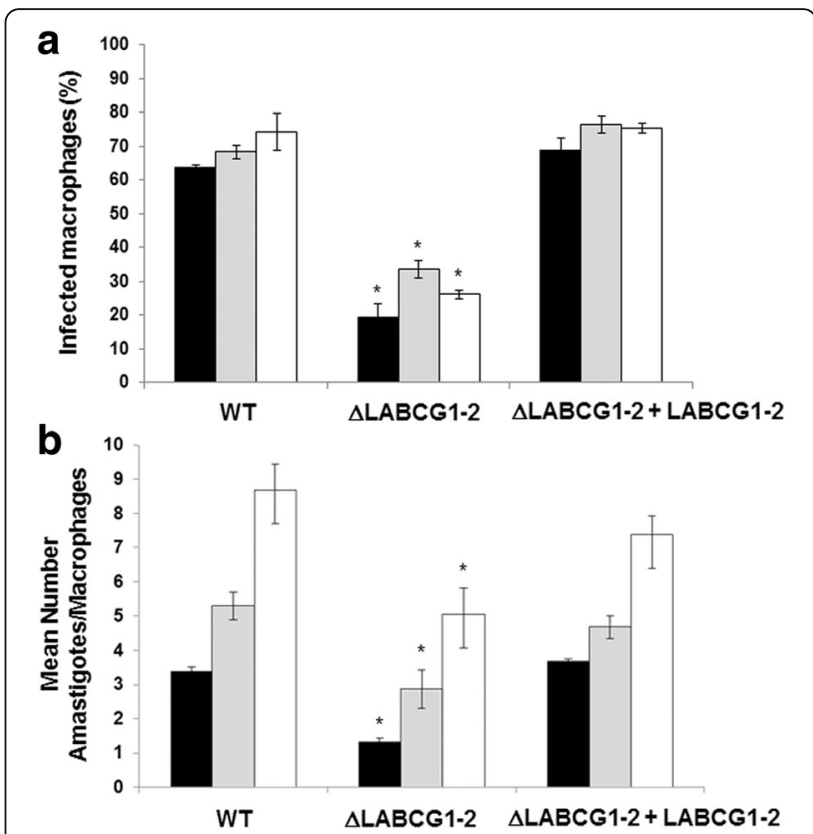

Fig. 2 Infectivity and survival of Leishmania lines in mouse peritoneal macrophages. Mouse peritoneal macrophages were infected with different L. major lines for $4 \mathrm{~h}$ at $35^{\circ} \mathrm{C}$. At 24 (black histograms), 72 (grey histograms) and $120 \mathrm{~h}$ (white histograms) post-infection, the infectivity \% (a) and the mean number of amastigotes/macrophages (b) were determined. Data are the mean \pm SD of three independent experiments. Significant differences versus the respective control line were determined using the Student's $t$-test $\left({ }^{*} P<0.05\right)$

susceptibility to human serum and expression of stagespecific protein HASPB. $\triangle$ LABCG1-2 parasites displayed a growth phenotype similar to the WT and add-back parasites in the usual culture medium (data not shown). Metacyclic parasites were purified on day five of the growth curve (stationary phase) by binding to the lectin PNA [30]. We observed that the percentage of non-agglutinated parasites $\left(\mathrm{PNA}^{-}\right)$was significantly lower in $\triangle \mathrm{LABCG} 1-2$ parasites $(1.3 \%)$ compared with WT $(17 \%)\left(t\right.$-test: $t_{(4)}=37.35, P$ $<0.0001)$ and add-back parasites (16\%) $\left(t\right.$-test: $t_{(4)}=20.78$, $P<0.0001$; Fig. 4a). This phenotype was not observed in the $L$. major line expressing a mutant version of LABCG2, probably because the wild-type version of LABCG2 in these parasites was not completely inactivated whereas the LABCG2 expression in $\triangle$ LABCG1-2 parasites was entirely suppressed.

Additionally, we analysed the susceptibility of the different Leishmania lines to lysis by human serum. As shown in Fig. 4b1, the different Leishmania lines in the logarithmic phase of growth presented a similar susceptibility to lysis by human serum; however, in the stationary growth phase, $\triangle$ LABCG1-2 parasites showed an increase in susceptibility to complement-mediated lysis at $10 \%$ human serum of around 2.5 -fold relative to the controls (Fig. 4b2). Serum concentrations higher than 


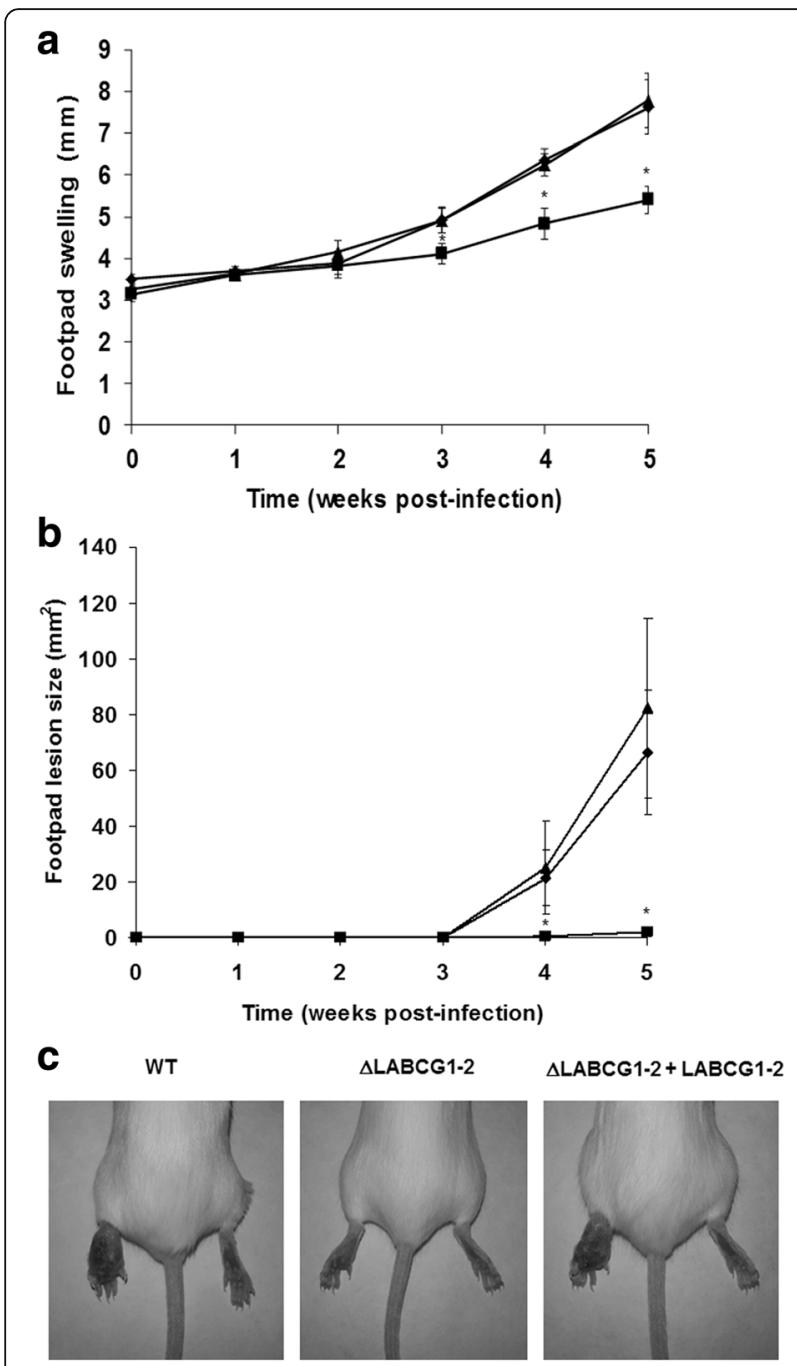

Fig. $3 \triangle L A B C G 1-2$ parasites are less infective in a mouse model of cutaneous leishmaniasis. Susceptible BALB/c mice were infected with $10^{5}$ WT (diamonds, black histograms), $\triangle \mathrm{LABCG} 1-2$ (squares, grey histograms) and add-back ( $\triangle \mathrm{LABCG} 1-2+\mathrm{LABCG} 1-2$; triangles, white histograms) L. major parasites in the stationary growth phase. Disease development was monitored weekly by measuring the footpad swelling (a) and lesion size (b). The pictures in c show the lesion at week 5 post-infection. The results represent the mean \pm SD of two independent experiments, with 6 mice per group. Mice were euthanized when the lesion size in controls reached a value of $60-80 \mathrm{~mm}^{2}$. ${ }^{*} P<0.05$ vs WT parasites

$10 \%$ were needed for a complete lysis of $\triangle$ LABCG1-2 parasites (Fig. 4b2); in contrast, only $50 \%$ of WT and add-back metacyclic parasites were lysed at the same serum concentration.

The only criterion supporting that $\triangle \mathrm{LABCG} 1-2$ parasites had displayed metacyclogenesis was a similar expression of metacyclic specific protein HASPB in the different stationary-phase promastigotes (Fig. 4c). A similar situation has been described in a $L$. major mutant VPS4 line involved in endosome sorting and autophagy [28]. In this case, the mutant parasites failed to differentiate into metacyclic forms as determined by the absence of HASPB and SHERP metacyclic specific proteins and a higher susceptibility to lysis by human serum [28]. However, the study did not observe any changes in the percentage of $\mathrm{PNA}^{-}$parasites. Our results concerning the unmodified expression of the HASPB protein in $\triangle \mathrm{LABCG} 1-2$ parasites established markers (although they lacked others) for metacyclic promastigotes indicating that the presence of the specific metacyclic protein HASPB was not enough to confer an infective phenotype. Probably, as suggested for the $L$. major mutant VPS4 line, the biosynthetic pathway of LPG assembly is induced independently of metacyclic specific proteins such as HASPB.

Thus, the reduced number of purified metacyclics, the higher susceptibility to complement-mediated lysis and the significant decrease in the infectivity of $\triangle$ LABCG1-2 parasites could be due to the influence of LABCG1-2 transporters in the appropriate expression of surface molecules (e.g. LPG) [31]. As previously shown, the complement resistance of $L$. major promastigotes strongly depends on LPG chain length and the formation of a thick protective glycocalyx surface [9].

In all Leishmania species, LPG is composed by an GPI anchor of a 1-O-alkyl-2-lysophosphatidylinositol lipid and a heptasaccharide core, to which is attached a long phosphoglycan (PG) polymer composed of 15-30 [Gal $\beta 1,4-$ Man $\left.\alpha 1-\mathrm{PO}_{4}\right]$ repeat units, playing a relevant role in parasite survival [9]. Some evidence suggests that LPG could decrease phagosome fusion properties at the first stages of infection in macrophages [32]. In this way, an attenuation of the virulence in promastigotes that lack LPG has previously been described using a $L$. major $\operatorname{lpg} 1^{-}$ mutant; and modifications in the LPG structures modulated phagosome-endosome fusion.

Using two different monoclonal antibodies against Leishmania LPG: (i) WIC79.3 (specific for terminal Gal ( $\beta 1-3)$ side-chains), and (ii) 3 F12 (specific for terminal arabinose in side chains of metacyclic promastigotes), we have observed differences in LPG composition in $\triangle \mathrm{LABCG} 1-2$ parasites since none of the antibodies employed recognised its LPG during the stationary growth phase compared with the WT and add-back lines (Fig. 4d). In previous experiments, we have shown that $\triangle \mathrm{LABCG} 1-2$ parasites were agglutinated with PNA, hence discarding the possibility of a lack of LPG. We hypothesise that LABCG1-2 could be involved in the intravesicular transport of oligosaccharides and the loss of LABCG1-2 could affect the LPG composition and the associated phenotypes. Future studies will aim to identify the type of oligosaccharide transported by LABCG1-2. 

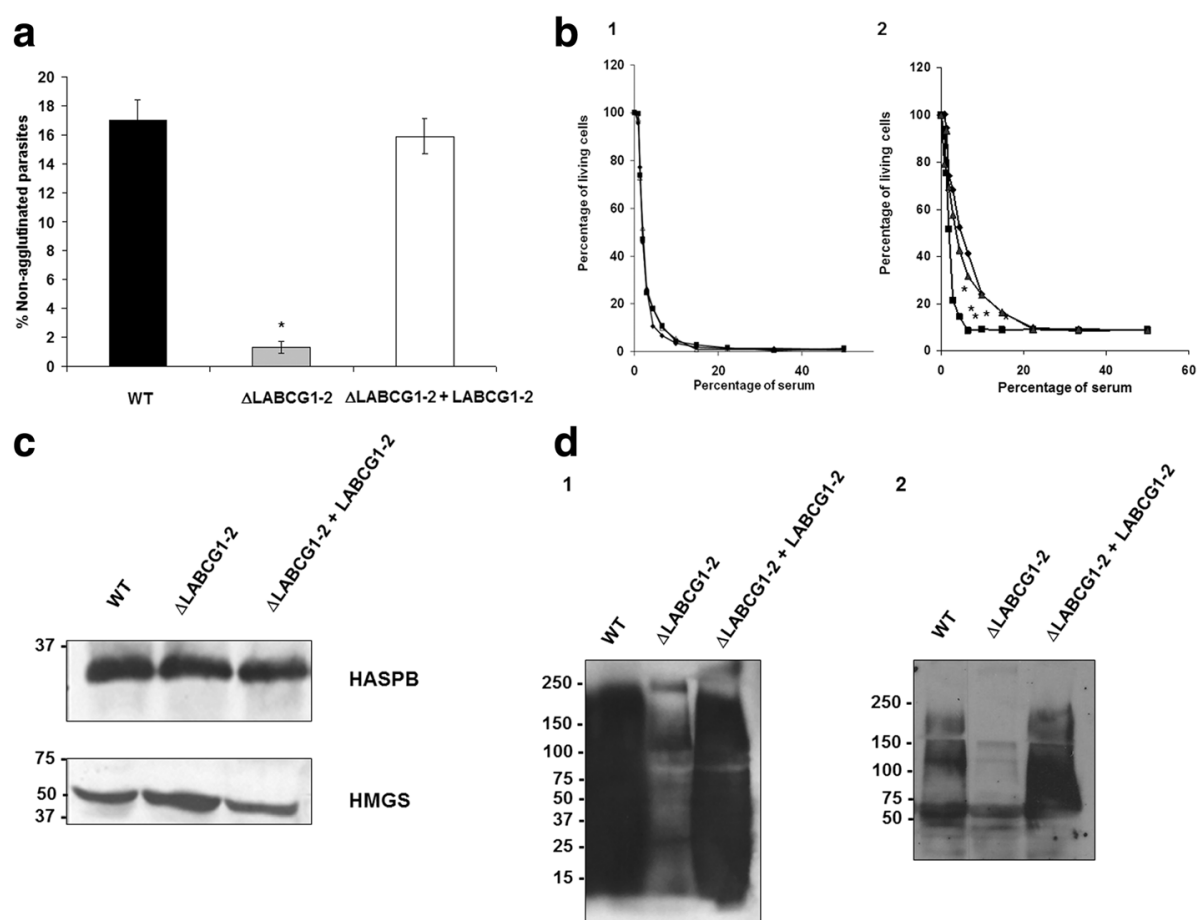

Fig. 4 Metacyclogenesis and Western blot analysis of LPG from different $L$. major lines. Proportion of metacyclic promastigotes of different $L$. major lines including: control (WT, black histograms, diamonds), $\triangle \mathrm{LABCG1-2}$ (grey histograms, squares) and add-back parasites ( $\triangle \mathrm{LABCG} 1-2+$ LABCG1-2, white histograms, triangles), in stationary phase of culture (day 5), assessed using the PNA assay (a). b Promastigotes $\left(1 \times 10^{7} / \mathrm{ml}\right)$ in the logarithmic (b1) or stationary phase (b2) of the growth curve were exposed to increasing concentrations of fresh human serum for 30 min at $37^{\circ} \mathrm{C}$. Resazurin was then added and the promastigotes incubated at $28^{\circ} \mathrm{C}$ for $24 \mathrm{~h}$ in order to determine cell viability. Data are the mean $\pm \mathrm{SD}$ of three independent experiments. Significant differences versus the respective control line were determined using the Student's $t$-test $\left({ }^{*} P<0.05\right)$. c Promastigotes $\left(1 \times 10^{7}\right)$ of WT, $\triangle L A B C G 1-2$ and $\triangle L A B C G 1-2+L A B C G 1-2$ were lysed and collected in the stationary growth phase and Western blot analysis with the antibodies HASPB and HMGS was performed. A Western blot assay representative of at least three independent experiments is shown. The positions of molecular markers $(\mathrm{kDa})$ are indicated on the left. $\mathbf{d}$ Promastigotes $\left(1 \times 10^{7}\right)$ of $\mathrm{WT}, \triangle \mathrm{LABCG} 1-2$ and $\triangle \mathrm{LABCG} 1-2+$ LABCG1-2 were collected at stationary phase of growth (8 days) and lysed as described in Materials and Methods. Western blot analysis of total proteins from parasites incubated with the antibodies WIC79.3 (d1) or 3 F12 (d2) were performed at 1:250 and 1:500 dilutions, respectively. A Western blot assay representative of at least three independent experiments is shown. The positions of molecular markers ( $k D a)$ are indicated on the left

\section{$\triangle \mathrm{LABCG} 1-2$ parasites are defective in autophagy}

Autophagy has been considered as a survival stress response to starvation conditions $[28,33]$. Considering that $\triangle$ LABCG1-2 parasites had altered the metacyclogenesis, we decided to determine whether these parasites would modify the autophagy as an important process for protein and organelle degradation during cellular differentiation and metacyclogenesis. Leishmania major ATG8 has been identified as an useful marker for observing autophagosomes in Leishmania [28]. Thus, to follow the formation of autophagosomes, we expressed ATG8 fused with RFP at its N-terminus in WT, $\triangle \mathrm{LABCG} 1-2$ and add-back parasites. Afterwards, the formation of autophagosomes was analyzed through the 10-day growth cycle of Leishmania lines (Fig. 5a). RFP-ATG8 was distributed throughout the cytoplasm in logarithmic-phase promastigotes; however, during the early stationary-phase, autophagosomes could be identified in some of the parasites as punctate structures in the cytoplasm [28]. The results show that the number of parasites containing autophagosomes increased from early log to early stationary-phase in WT and addback promastigotes, with a maximum at day 8 (Fig. 5a). However, in $\triangle \mathrm{LABCG1-2}$ parasites, the percentage of cells with autophagosomes was significantly lower at the different time-points ( $t$-test: $t_{(4)}=8.10-24.74, \quad P=0.0001-$ 0.0013; Fig. 5a). When parasites progressed to stationary phase and differentiate into metacyclic forms, the percentage of cells with autophagosomes rapidly decreased at days 9-10 (Fig. 5a). These results were validated by a well established Western blot assay using anti-RFP antibody on cell extracts from WT, $\triangle \mathrm{LABCG} 1-2$ and add-back lines expressing RFP-ATG8, in order to ascertain the proportion of cytosolic and lipid-associated forms of ATG8-PE (Fig. 5b) [12, 28]. The lipidated bands (RFP-ATG8-PE) associated with the autophagosomal membrane migrate faster than the unlipidated bands (RFP-ATG8). In WT and add-back lines at a stationary phase of growth, the proportion of RFP-ATG8-PE increased significantly versus the 


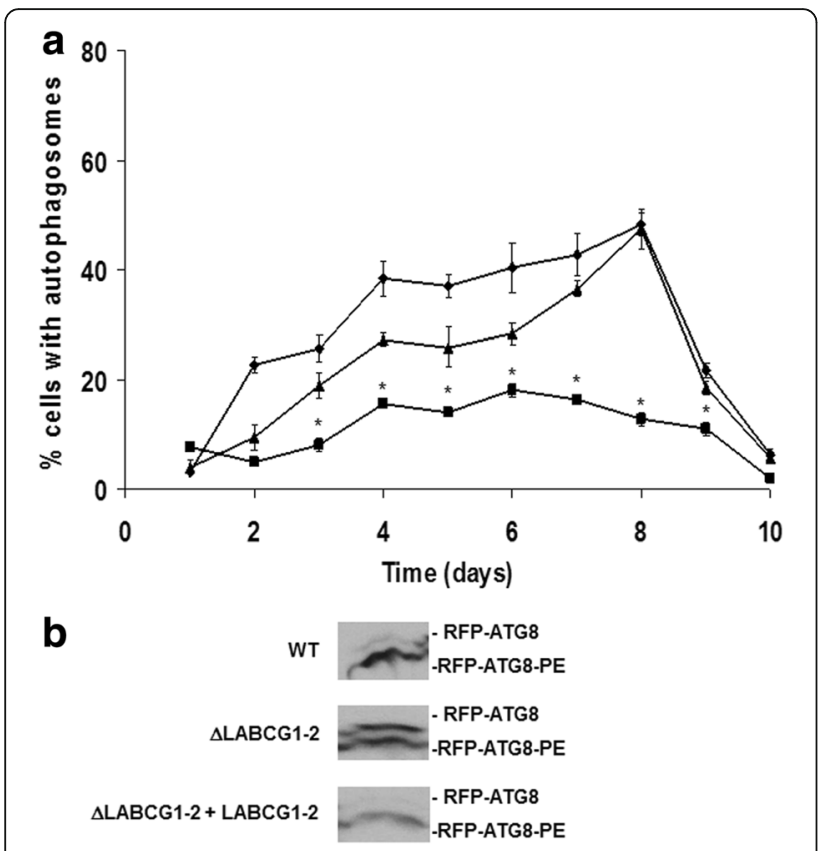

Fig. 5 Autophagosome formation in promastigotes of L. major lines. a The proportion of promastigotes from different lines including: control (WT) (diamonds), $\triangle \mathrm{LABCG} 1-2$ (squares), and $\triangle \mathrm{LABCG} 1-2+\mathrm{LABCG} 1-2$ (triangles) expressing RFP-ATG8 was assessed during the growth curve in vitro. Data are the mean \pm SD from three independent experiments. Statistical differences relative to the WT values were determined using the Student's $t$ test $\left.{ }^{*} P<0.05\right)$. b Western blot analysis of RFP-ATG8 in different Leishmania lines. Promastigote cell extracts from different lines (WT, $\triangle$ LABCG1-2 and $\triangle$ LABCG1-2 + LABCG1-2) transfected with RFPATG8 were collected during the stationary growth phase, separated by SDS-PAGE in the presence of $6 \mathrm{M}$ urea and analysed by Western blot. A Western blot assay representative of at least three independent experiments is shown

unlipidated form (Fig. 5b). In contrast, $\triangle$ LABCG1-2 parasites had a higher proportion of non-lipidated ATG8 versus WT and add-back lines (Fig. 5b), which is consistent with a decrease in autophagosome biogenesis. These data clearly suggest that $\triangle$ LABCG1-2 parasites have an autophagy defect in which autophagosome formation is inhibited, increasing the susceptibility to nutrient starvation and consequently impairing the metacyclogenesis.

A defect in autophagy progress has been described in $L$. major VPS4 and ATG4.2 mutant lines involved in endosome sorting and autophagy [28]; the authors concluded that differentiation to metacyclic forms in Leishmania is dependent on endosome function and autophagy.

\section{Increased ROS levels in LABCG1-2 null mutant parasites}

The relationships between ROS levels and autophagy induction previously described in mammalian cells and Leishmania [12, 34], prompted us to study whether $\triangle$ LABCG1-2 parasites present a modification in ROS levels during the parasite's different growth cycle phases. ROS levels in WT and add-back Leishmania lines increased through the growth phases from early log phase to mid log and early stationary phases, but subsequently diminished when the cells entered the stationary phase (Fig. 6, time-points equivalent to 2, 4, 6 and 9 days, respectively). However, in $\triangle \mathrm{LABCG} 1-2$ parasites, ROS levels were 2-fold higher than their control parasites (WT) at the early stationary and stationary phases of growth (Fig. 6); this phenotype was re-established to WT levels in the add-back parasites (Fig. 6). Thus, the period at which ROS levels were highest agrees with the moment when autophagy was significantly reduced (Fig. 5a). Similar findings have been reported in L. major null mutant for ATG4.2 cysteine peptidase, which, although it presents a significant increase in ROS levels at different growth phases (mainly at the stationary phase), was unable to form autophagosomes under starvation conditions [12]. These results support previous findings [12] suggesting that autophagy is important for the protection of parasites against ROS-mediated protein damage and its absence results in elevated ROS levels.

\section{An increase in thiols influences autophagy response in $\triangle \mathrm{LABCG} 1-2$ parasites}

Redox imbalance has a key role in driving autophagy [35]. In eukaryotic cells, a decrease in the GSH/GSSG ratio due to a release of GSH to the extracellular milieu mediated by $\mathrm{ABCC} 1$ and the concomitant increase of oxidized thiols induces autophagy [14]. Evidence linking thiol redox state with autophagy has been reported previously [35]. The hypothesis does not exclude the possibility that redox imbalance might regulate autophagy at multiple levels. It has recently been demonstrated that nutrient deprivation in carcinoma cells led to a significant decrease

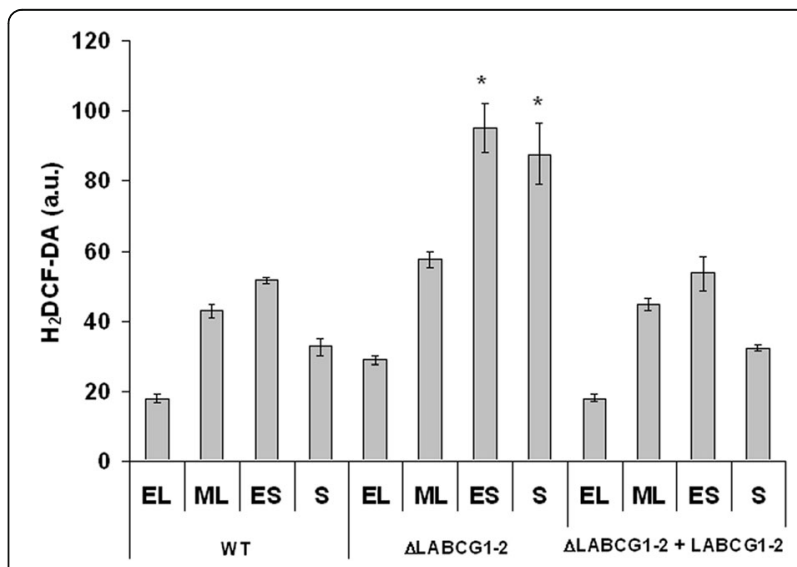

Fig. 6 ROS levels along the growth cycle of Leishmania lines. Promastigotes $\left(1 \times 10^{7}\right)$ were collected at early logarithmic (EL), mid logarithmic (ML), early stationary (ES) and stationary (S) phases of growth (2, 4, 6 and 10 days, respectively) and basal ROS levels were measured by means of an $\mathrm{H}_{2} \mathrm{DCFDA}$ assay. Data are the mean $\pm \mathrm{SD}$ of three independent experiments. Significant differences versus the respective control line were determined using Student's $t$-test $\left({ }^{*} P<0.05\right)$ 
of intracellular GSH levels and an activation of autophagy [14]. In Leishmania parasites, the LABCG2 transporter has been described as a non-protein thiol transporter [19]. In fact, we have found evidence that $\triangle$ LABCG1-2 parasites have modified intracellular thiols. No differences in the parasite size that could explain the intracellular thiol variance was observed between the different lines (data not shown). As depicted in Fig. 7a, a significant increase in thiol levels was observed in $\triangle$ LABCG1-2 parasites versus the control (WT) $\left(t\right.$-test: $\left.t_{(4)}=47.90, P<0.0001\right)$ and add-back parasites $\left(t\right.$-test: $\left.t_{(4)}=93.60, P<0.0001\right)$; this increase in thiols correlates with a low proportion of cells containing autophagosomes (Fig. 7b), supporting the idea that Leishmania LABCG1-2 transporters are involved in autophagy and influence intracellular thiol levels. To assess the specificity of these results we measured intracellular thiol levels in the presence of GSH synthesis inhibitor BSO [36]. As shown in Fig. 7a, treatment with BSO

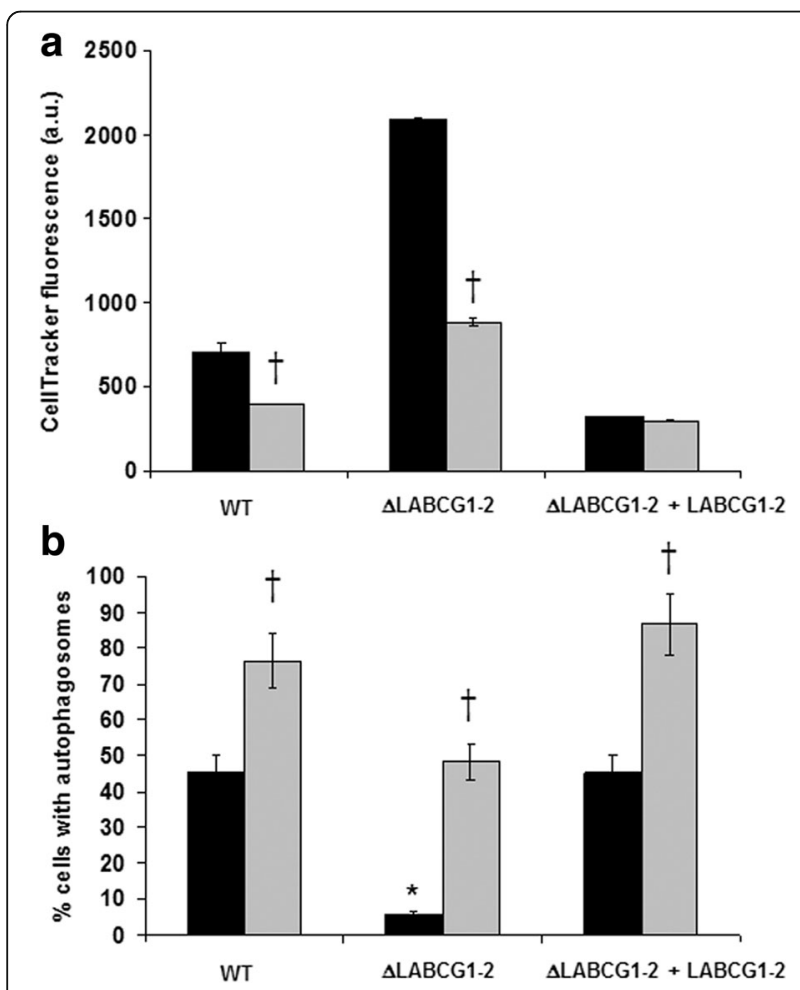

Fig. 7 Relationship between thiol levels and autophagosome formation in L. major lines. a Parasites (WT, $\triangle \mathrm{LABCG} 1-2$ and $\triangle \mathrm{LABCG} 1-2+\mathrm{LABCG} 1-$ 2) transfected with pNUS RFP-ATG8 were pre-incubated for $48 \mathrm{~h}$ in $\mathrm{M}$ 199 without (black histograms) or with (grey histograms) 3 mM BSO in order to deplete thiol levels. After 8 days in culture, promastigotes $(4 \times$ $10^{6}$ ) were collected and incubated with $2 \mu \mathrm{M}$ CellTracker ${ }^{\mathrm{TM}}$ for $15 \mathrm{~min}$. Fluorescence intensities were measured by flow cytometry. $\mathbf{b}$ In parallel, we measured the proportion of promastigotes expressing RFP-ATG8 with (grey histograms) or without (black histograms) BSO treatment by counting autophagosomes using a fluorescence microscope. Data are the mean $\pm S D$ from three independent experiments. Significant differences were determined by the Student's $t$-test $\left({ }^{*} P<0.01\right.$ versus $W T ;+P<0.01$ versus non-treated parasites) produces a decrease of GSH levels in WT and $\triangle \mathrm{LABCG1-}$ 2 lines. However, we did not observe a significant decrease in the add-back line, probably due to a slight overexpression of LABCG1-2 that could transport the CellTracker ${ }^{\text {tix }}$ probe, which was previously shown to be transported by human ABCG2 [37]. To evaluate whether the decrease in thiols was associated with autophagosome generation, we determined autophagosome formation in the presence or absence of BSO. Figure $7 \mathrm{~b}$ shows that $\mathrm{BSO}$ preincubation for $48 \mathrm{~h}$ caused a decrease of intracellular GSH levels,



Fig. 8 Reduced non-protein thiol levels in L. major lines. a Parasites $\left(3 \times 10^{8}\right)$ were collected from different lines: WT (black histograms), $\triangle \mathrm{LABCG} 1-2$ (grey histograms), and $\triangle \mathrm{LABCG} 1-2+\mathrm{LABCG} 1-2$ (white histograms) at stationary-phase growth. After three washes with PBS, the cell pellet was resuspended in Tris-HCl 10 mM-EDTA supplemented with 2\% Triton X-100 and thiol levels quantified by flow cytometry. $\mathbf{b}$ Promastigotes $\left(1 \times 10^{9}\right)$ from different lines were collected, lyophilised and stored at $4{ }^{\circ} \mathrm{C}$ until used. The cell pellet was treated as described in Materials and Methods. Data are the mean \pm SD from three independent experiments. Significant differences versus WT parasites were determined with the Student's $t$-test $(* P<0.01)$ 
while significantly increasing the percentage of cells containing autophagosomes in all Leishmania lines $\left(t\right.$-test: $t_{(4)}$ $=8.38, P=0.0011$ for WT; $t$-test: $t_{(4)}=20.82, P<0.0001$ for $\triangle$ LABCG1-2; $t$-test: $t_{(4)}=11.03, \quad P=0.0004$ for $\triangle \mathrm{LABCG} 1-2+\mathrm{LABCG} 1-2)$. These results provide evidence that autophagosome formation in Leishmania is directly linked to intracellular thiol levels and that a decrease in thiols stimulates the autophagy. Collectively, this suggests that a decrease in thiols is a specific feature of autophagy and that LABCG1-2 transporters are involved in this process. The relationship between autophagy regulation and GSH transport has been suggested previously for human ABCG2 transporter [15].

We have quantified the levels of thiols in the different Leishmania lines using DTNB probe, and the results show that $\triangle \mathrm{LABCG} 1-2$ parasites present significantly higher levels of non-protein thiols (2.2-fold increase) versus those observed in WT $\left(t\right.$-test: $\left.t_{(4)}=17.30, P<0.0001\right)$ and addback parasites ( $t$-test: $t_{(4)}=25.29, P<0.0001$ ) (Fig. $8 \mathrm{a}$ ). Additionally, we have determined by HPLC (Fig. $8 \mathrm{~b}$ ) that $\mathrm{GSH}$ and $\mathrm{T}[\mathrm{SH}]_{2}$ were the main molecules that differentially accumulated into $\triangle$ LABCG1-2 parasites, around 2fold for GSH and 1.5-fold for $\mathrm{T}[\mathrm{SH}]_{2}$ compared with WT ( $t$-test: $t_{(6)}=3.91, P=0.0078$ for GSH; $t$-test: $t_{(6)}=$ 4.22, $P=0.0055$ for $\left.\mathrm{T}[\mathrm{SH}]_{2}\right)$ and add-back parasites $(t$ test: $t_{(6)}=4.76, P=0.0031$ for GSH; $t$-test: $t_{(6)}=10.72, P<$ 0.0001 for $\mathrm{T}[\mathrm{SH}]_{2}$ ) (Fig. 8b).

Future studies using membranes from baculovirusinsect cell heterologous expression of LABCG1-2 will confirm their involvement in non-protein thiols transport.

\section{Conclusions}

In conclusion, the Leishmania LABCG1-2 transporters could be considered as GSH and T $[\mathrm{SH}]_{2}$ thiol transporters, as they can modulate the levels of these molecules into the parasites influencing the autophagy process. Our finding that LABCG1-2 expression is associated with stress-induced autophagy indicates that these transporters have a novel role beyond drug-resistance, PS transport and virulence. LABCG1-2 transporters could interact with other protein (s) and the consequently alteration of these interactions may account for some of the observed phenotypes.

\section{Abbreviations \\ ABC: ATP-binding cassette; DIG: Digoxigenin; HASPB: Hydrophilic acylated surface protein B; HMGS: 3-Hydroxy-3-methyl-glutaryl Coenzyme A synthase; HPLC: High performance liquid chromatography; Kb: Kilobase; Kbp: Kilobase pair; kDa: KiloDalton; LPG: Lipophosphoglycan; PBS: Phosphate buffered saline; PCR: Polymerase chain reaction; PS: Phosphatidylserine; RFP: Red fluorescent protein; ROS: Reactive oxygen species; WT: Wild-type}

\section{Acknowledgements}

We thank M. Delgado and E. González-Rey for help with in vivo animal experiments, J. Mottram (University of Glasgow, UK) for the plasmid pNUS ATG8-RFP used in this research work and S.M. Beverley (Washington University, USA) for providing the pIRSAT vector used throughout this research work. Also, we thank G. Spaeth (Institute Pasteur, Paris) for the antibody WIC 73.9, D. Sacks (NIH/NIAID) for the antibody 3 F12, D. Smith (University of York, UK) for the anti-
HASPB antibody and D. Gonzalez-Pacanowska and L.M. Ruiz-Perez (IPBLN-CSIC, Granada, Spain) for the LmHMGS antibody.

\section{Funding}

This work was supported by the Spanish Grants SAF2015-68042-R (to S.C. and F.G.), SAF2012-34267 (to F.G.), by the Proyecto de Excelencia, Junta de Andalucia, Ref. CTS-7282 (to F.G.) and by FEDER funds from the EU to S.C. and F.G. A. Perea was a student of the PhD program "Biochemistry and Molecular Biology" of the University of Granada (Spain) and was supported by a fellowship for predoctoral contracts for PhD training from the Ministerio de Economia y Competitividad (in charge of Project SAF2012-34267).

\section{Availability of data and materials}

The datasets and analysed data during the current study are available from the corresponding author on reasonable request.

\section{Authors' contributions}

FG and SC designed the study and analyzed the results. JIM and AP developed the virulence, autophagy and oxidative stress related experiments. DL generated the null mutant $\triangle$ LABCG1-2 parasites. JC was involved in infectivity and metacyclogenesis assays. LP determined the non-protein thiols by HPLC. JIM and FG wrote the manuscript. All authors read and approved the final manuscript.

\section{Competing interests}

The authors declare that they have no competing interests.

\section{Consent for publication}

Not applicable.

\section{Ethics approval and consent to participate}

All experiments involving the use of laboratory animals were performed according to National/EU guidelines regarding the care and use of laboratory animals in research. Approval for these studies was obtained from the Ethics Committee of the Spanish National Research Council (CSIC file CEA-213-1-11).

\section{Publisher's Note}

Springer Nature remains neutral with regard to jurisdictional claims in published maps and institutional affiliations.

\section{Author details}

${ }^{1}$ Instituto de Parasitología y Biomedicina "López-Neyra", IPBLN-CSIC, Parque Tecnológico de Ciencias de la Salud, Avda. del Conocimiento s/n, 18016 Granada, Spain. ${ }^{2}$ Departamento de Bioquímica, Facultad de Medicina, Universidad de la República, Montevideo, Uruguay.

Received: 7 December 2016 Accepted: 15 May 2017

Published online: 30 May 2017

\section{References}

1. Alvar J, Velez ID, Bern C, Herrero M, Desjeux P, Cano J, Jannin J, Den Boer M. Leishmaniasis worldwide and global estimates of its incidence. PLOS ONE. 2012;7(5), e35671.

2. Higgins CF. ABC transporters: from microorganisms to man. Annu Rev Cell Biol. 1992:8:67-113.

3. Leprohon P, Legare D, Girard I, Papadopoulou B, Ouellette M. Modulation of Leishmania $A B C$ protein gene expression through life stages and among drug-resistant parasites. Eukaryot Cell. 2006;5(10):1713-25.

4. Manzano Jl, Garcia-Hernandez R, Castanys S, Gamarro F. A new ABC halftransporter in Leishmania major is involved in resistance to antimony. Antimicrob Agents Chemother. 2013;57(8):3719-30.

5. Bates SE, Robey R, Miyake K, Rao K, Ross DD, Litman T. The role of halftransporters in multidrug resistance. J Bioenerg Biomembr. 2001;33(6):503-11.

6. Campos-Salinas J, Leon-Guerrero D, Gonzalez-Rey E, Delgado M, Castanys S, Perez-Victoria JM, Gamarro F. LABCG2, a new ABC transporter implicated in phosphatidylserine exposure, is involved in the infectivity and pathogenicity of Leishmania. PLoS Negl Trop Dis. 2013;7(4), e2179.

7. Ouakad M, Vanaerschot M, Rijal S, Sundar S, Speybroeck N, Kestens L, et al. Increased metacyclogenesis of antimony-resistant Leishmania donovani clinical lines. Parasitology. 2011;138(11):1392-99. 
8. Saraiva EM, Pinto-da-Silva LH, Wanderley JL, Bonomo AC, Barcinski MA Moreira ME. Flow cytometric assessment of Leishmania spp. metacyclic differentiation: validation by morphological features and specific markers. Exp Parasitol. 2005;110(1):39-47.

9. Spath GF, Garraway LA, Turco SJ, Beverley SM. The role(s) of lipophosphoglycan $(\mathrm{LPG})$ in the establishment of Leishmania major infections in mammalian hosts. Proc Natl Acad Sci USA. 2003;100(16):9536-41.

10. Lodge R, Diallo TO, Descoteaux A. Leishmania donovani lipophosphoglycan blocks NADPH oxidase assembly at the phagosome membrane. Cell Microbiol. 2006;8(12):1922-31

11. Franco LH, Beverley SM, Zamboni DS. Innate immune activation and subversion of mammalian functions by Leishmania lipophosphoglycan. J Parasitol Res. 2012; 2012:165126

12. Williams RA, Mottram JC, Coombs GH. Distinct roles in autophagy and importance in infectivity of the two ATG4 cysteine peptidases of Leishmania major. J Biol Chem. 2013;288(5):3678-90

13. Deffieu M, Bhatia-Kissova I, Salin B, Galinier A, Manon S, Camougrand N. Glutathione participates in the regulation of mitophagy in yeast. J Biol Chem. 2009;284(22):14828-37.

14. Desideri E, Filomeni G, Ciriolo MR. Glutathione participates in the modulation of starvation-induced autophagy in carcinoma cells. Autophagy. 2012;8(12): 1769-81.

15. Ding $\mathrm{R}$, Jin $\mathrm{S}$, Pabon $\mathrm{K}$, Scotto KW. A role for ABCG2 beyond drug transport: Regulation of autophagy. Autophagy. 2016;12(5):737-51.

16. Ballatori N, Krance SM, Marchan R, Hammond CL. Plasma membrane glutathione transporters and their roles in cell physiology and pathophysiology. Mol Aspects Med. 2009;30(1-2):13-28.

17. Lee JY, Yang JG, Zhitnitsky D, Lewinson O, Rees DC. Structural basis for heavy metal detoxification by an Atm1-type ABC exporter. Science. 2014; 343(6175):1133-6.

18. Gauthier C, Ozvegy-Laczka C, Szakacs G, Sarkadi B, Di Pietro A. ABCG2 is not able to catalyze glutathione efflux and does not contribute to GSH-dependent collateral sensitivity. Front Pharmacol. 2013:4:138.

19. Perea A, Manzano Jl, Castanys S, Gamarro F. The LABCG2 Transporter from the protozoan parasite Leishmania is involved in antimony resistance. Antimicrob Agents Chemother. 2016;60(6):3489-96.

20. Perez-Victoria FJ, Sanchez-Cañete MP, Castanys S, Gamarro F. Phospholipid translocation and miltefosine potency require both $L$. donovani miltefosine transporter and the new protein LdRos3 in Leishmania parasites. J Biol Chem. 2006:281(33):23766-75.

21. Garcia-Sanchez S, Sanchez-Cañete MP, Gamarro F, Castanys S. Functional role of evolutionarily highly conserved residues, $\mathrm{N}$-glycosylation level and domains of the Leishmania miltefosine transporter-Cdc50 subunit. Biochem J. 2014;459(1):83-94.

22. Lira R, Doherty M, Modi G, Sacks D. Evolution of lesion formation, parasitic load, immune response and reservoir potential in C57BL/6 mice following high- and low-dose challenge with Leishmania major. Infect Immun. 2000; 68(9):5176-82

23. Sacks DL, Hieny S, Sher A. Identification of cell surface carbohydrate and antigenic changes between noninfective and infective developmental stages of Leishmania major promastigotes. J Immunol. 1985;135(1):564-9.

24. Kelleher M, Bacic A, Handman E. Identification of a macrophage-binding determinant on lipophosphoglycan from Leishmania major promastigotes. Proc Natl Acad Sci USA. 1992:89(1):6-10.

25. Kelleher M, Curtis JM, Sacks DL, Handman E, Bacic A. Epitope mapping of monoclonal antibodies directed against lipophosphoglycan of Leishmania major promastigotes. Mol Biochem Parasitol. 1994:66(2):187-200.

26. Flinn HM, Rangarajan D, Smith DF. Expression of a hydrophilic surface protein in infective stages of Leishmania major. Mol Biochem Parasitol. 1994; 65(2):259-70.

27. Carrero-Lerida J, Perez-Moreno G, Castillo-Acosta VM, Ruiz-Perez LM, Gonzalez-Pacanowska D. Intracellular location of the early steps of the isoprenoid biosynthetic pathway in the trypanosomatids Leishmania major and Trypanosoma brucei. Int J Parasitol. 2009;39(3):307-14

28. Besteiro S, Williams RA, Morrison LS, Coombs GH, Mottram JC. Endosome sorting and autophagy are essential for differentiation and virulence of Leishmania major. J Biol Chem. 2006;281(16):11384-96.

29. Krauth-Siegel RL, Jacoby EM, Schirmer RH. Trypanothione and N1glutathionylspermidine: isolation and determination. Methods Enzymol. 1995:251:287-94.
30. Spath GF, Beverley SM. A lipophosphoglycan-independent method for isolation of infective Leishmania metacyclic promastigotes by density gradient centrifugation. Exp Parasitol. 2001;99(2):97-103.

31. Turco SJ, Descoteaux A. The lipophosphoglycan of Leishmania parasites. Annu Rev Microbiol. 1992:46:65-94.

32. Dermine JF, Scianimanico S, Prive C, Descoteaux A, Desjardins M. Leishmania promastigotes require lipophosphoglycan to actively modulate the fusion properties of phagosomes at an early step of phagocytosis. Cell Microbiol. 2000;2(2):115-26.

33. Levine B, Klionsky DJ. Development by self-digestion: molecular mechanisms and biological functions of autophagy. Dev Cell. 2004;6(4):463-77.

34. Wong CH, Iskandar KB, Yadav SK, Hirpara JL, Loh T, Pervaiz S. Simultaneous induction of non-canonical autophagy and apoptosis in cancer cells by ROS-dependent ERK and JNK activation. PLoS One. 2010;5(4):e9996.

35. Filomeni G, Desideri E, Cardaci S, Rotilio G, Ciriolo MR. Under the ROS...thio network is the principal suspect for autophagy commitment. Autophagy. 2010;6(7):999-1005.

36. El Fadili K, Messier N, Leprohon P, Roy G, Guimond C, Trudel N, et al. Role of the $A B C$ transporter MRPA (PGPA) in antimony resistance in Leishmania infantum axenic and intracellular amastigotes. Antimicrob Agents Chemother. 2005;49(5):1988-93.

37. Strouse JJ, Ivnitski-Steele I, Waller A, Young SM, Perez D, Evangelisti AM, et al. Fluorescent substrates for flow cytometric evaluation of efflux inhibition in ABCB1, ABCC1, and ABCG2 transporters. Anal Biochem. 2013:437(1):77-87.

\section{Submit your next manuscript to BioMed Central and we will help you at every step:}

- We accept pre-submission inquiries

- Our selector tool helps you to find the most relevant journal

- We provide round the clock customer support

- Convenient online submission

- Thorough peer review

- Inclusion in PubMed and all major indexing services

- Maximum visibility for your research

Submit your manuscript at www.biomedcentral.com/submit

) Biomed Central 